\title{
The Association Between Receipt of Guideline-Concordant Long-Term Opioid Therapy and All-Cause Mortality
}

\author{
Julie R. Gaither, PhD, MPH, RN ${ }^{1,2,3,4}$, Joseph L. Goulet, PhD',5, William C. Becker, MD2,6, \\ Stephen Crystal, PhD' ${ }^{7}$ E. Jennifer Edelman, MD, MHS ${ }^{4,6}$, Kirsha Gordon, MPhil, MS', \\ Robert D. Kerns, PhD ${ }^{2,5}$, David Rimland, MD ${ }^{8,9}$, Melissa Skanderson, MSW' \\ Amy C. Justice, $M D, P h D^{7,2,3,6}$, and David A. Fiellin, $M D^{7,4,6}$
}

\begin{abstract}
${ }^{1}$ Yale School of Public Health, Yale University, New Haven, CT, USA; ${ }^{2}$ VA Connecticut Healthcare System, West Haven, CT, USA; ${ }^{3}$ Yale Center for Medical Informatics, Yale School of Medicine, Yale University, New Haven, CT, USA; ${ }^{4}$ Center for Interdisciplinary Research on AIDS, Yale School of Public Health, Yale University, New Haven, CT, USA; ${ }^{5}$ Department of Psychiatry, Yale School of Medicine, Yale University, New Haven, CT, USA; ${ }^{6}$ Department of Internal Medicine, Yale School of Medicine, Yale University, New Haven, CT, USA; ${ }^{7}$ Institute for Health, Health Care Policy, and Aging Research, Rutgers University, New Brunswick, NJ, USA; ' Division of Infectious Diseases, Emory University School of Medicine, Atlanta, GA, USA; ${ }^{9}$ Atlanta VA Medical Center, Decatur, GA, USA; ${ }^{10}$ VA Pittsburgh Healthcare System, Pittsburgh, PA, USA.
\end{abstract}

PURPOSE: For patients receiving long-term opioid therapy (LtOT), the impact of guideline-concordant care on important clinical outcomes-notably mortality-is largely unknown, even among patients with a high comorbidity and mortality burden (e.g., HIV-infected patients). Our objective was to determine the association between receipt of guideline-concordant LtOT and 1-year all-cause mortality.

METHODS: Among HIV-infected and uninfected patients initiating LtOT between 2000 and 2010 through the Department of Veterans Affairs, we used Cox regression with time-updated covariates and propensity-score matched analyses to examine the association between receipt of guideline-concordant care and 1-year all-cause mortality. RESULTS: Of 17,044 patients initiating LtOT between 2000 and 2010, 1048 patients (6\%) died during 1 year of follow-up. Patients receiving psychotherapeutic cointerventions (hazard ratio [HR] 0.62; 95\% confidence interval [CI] $0.51-0.75 ; P<0.001)$ or physical rehabilitative therapies (HR 0.81; 95\% CI 0.67-0.98; $P=0.03$ ) had a decreased risk of all-cause mortality compared to patients not receiving these services, whereas patients prescribed benzodiazepines concurrent with opioids had a higher risk of mortality (HR 1.39; 95\% CI 1.12-1.66; $P<0.001$ ). Among patients with a current substance use disorder (SUD), those receiving SUD treatment had a lower risk of mortality than untreated patients (HR 0.47; 95\% CI 0.32$0.68 ; P=<0.001)$. No association was found between allcause mortality and primary care visits (HR 1.12; 95\% CI 0.90-1.26; $P=0.32$ ) or urine drug testing (HR 0.96; 95\% CI $0.78-1.17 ; P=0.67)$.

CONCLUSIONS: Providers should use caution in initiating LtOT in conjunction with benzodiazepines and untreated SUDs. Patients receiving LtOT may benefit from multi-modal treatment that addresses chronic pain and its associated comorbidities across multiple disciplines.

Electronic supplementary material The online version of this article (doi:10.1007/s11606-015-3571-4) contains supplementary material, which is available to authorized users.

Published online February 3, 2016
KEY WORDS: Opioid analgesics; practice guideline; quality of health care; mortality; pain.

J Gen Intern Med 31(5):492-501

DOI: $10.1007 / \mathrm{s} 11606-015-3571-4$

(C) Society of General Internal Medicine 2016

$\mathrm{O}$ pioid analgesics, medications once primarily prescribed in palliative care and postoperative settings, are now widely prescribed for chronic pain in specialty and general medical settings. ${ }^{1}$ Yet despite the risks posed by opioids, including the risk of addiction and overdose, long-term opioid therapy (LtOT; typically defined as receipt of opioids for $\geq 90$ days $)^{2-6}$ is rarely provided in accordance with clinical practice guidelines. ${ }^{3,7-16}$ Among the potential barriers to the provision of guideline-concordant LtOT are ambiguity and disagreements over which recommendations are important to patient outcomes and how they should be prioritized given time constraints and the competing demands of patient care. ${ }^{17-}$ ${ }^{19}$ The majority of recommendations promulgated by leading medical societies, including the American Pain Society and American Academy of Pain Medicine, ${ }^{3,7}$ are supported by low-quality evidence, and none are supported by evidence deemed to be of high quality. ${ }^{3,9,19,20}$ In 2009 , a multidisciplinary expert panel identified 37 key areas pertaining to patient care and LtOT where critical weaknesses in the literature exist ${ }^{19}$ to date, the majority of these gaps in the literature have gone unaddressed. ${ }^{19,20}$ Because it remains unclear which recommendations offer the greatest benefit to patient outcomes, clinicians may be relying on anecdotal evidence and individual experience when determining which, if any, LtOT recommendations to implement. ${ }^{17,18}$

Therefore, with the overarching goal of contributing to an evidence base to guide future quality improvement efforts targeting opioid-related adverse events, we examined the association between guideline-concordant LtOT and all-cause 
mortality using the Veterans Aging Cohort Study (VACS), a well-established, validated sample ${ }^{21-24}$ of HIV-infected patients demographically matched (1:2) to uninfected patients engaged in care. VACS researchers have previously reported that opioid receipt is common in this sample, and that HIVinfected patients are more likely to receive high-dose prescriptions ( $\geq 120$ milligrams morphine equivalents $[\mathrm{mEq}]$ per day). ${ }^{6}$ Thus, the VACS provides a robust sample in which to explore mortality associated with guideline-concordant LtOT.

We examined the impact of guideline-concordant LtOT on all-cause mortality in patients at the beginning stage of LtOT, a critical time when guideline-concordant care may be most important, ${ }^{3}$ and clinicians may thus be more diligent in delivering higher-quality care. ${ }^{25}$

\section{METHODS}

\section{Study Overview}

In a large sample of outpatients initiating LtOT between 2000 and 2010, we examined the association between receipt of guideline-concordant LtOT and 1-year all-cause mortality. To address the potential for confounding by indication, ${ }^{26,27}$ we examined these associations using a propensity-matched design.

\section{Data Source}

We abstracted administrative, clinical, laboratory, and pharmacy data from the Department of Veteran Affairs (VA) electronic medical record system for patients participating in the VACS. As previously described, ${ }^{21-24,28}$ VACS is a prospective cohort of HIV-infected patients matched $(1: 2)$ by age, sex, race, and VA site of care to uninfected controls. ${ }^{29}$ VACS is HIPAA compliant and has received approval from the review boards for the VA Connecticut Healthcare System and the Yale School of Medicine; the requirement for informed consent was waived.

\section{Study Population}

From the VA Pharmacy Benefits Management database, we captured outpatient prescriptions for oral and transdermal opioids filled or refilled between October 1, 1999 (fiscal year 2000) and September 30, 2010 (fiscal year 2010). Consistent with prior studies, we defined LtOT as receipt of at least a 90-day supply of opioids. ${ }^{2,4,6,16}$ We allowed for no more than a 30-day window between prescription refills. Finally, because our focus was on chronic pain, we excluded data on methadone and buprenorphine prescribed for opioid use disorder, but retained data on methadone prescribed for the treatment of pain. ${ }^{6}$

From the approximately 120,000 patients enrolled in the VACS (as of fiscal year 2012), ${ }^{29}$ as detailed in Figure 1, we identified 26,931 patients filling or refilling prescriptions for 90 or more days of opioids as outpatients between 2000 and
2010. We excluded from this cohort those filling opioid prescriptions within the prior 90 days in order to focus on incident versus prevalent cases of LtOT. We also excluded patients with fewer than 90 days of follow-up, including those who did not meet the 90-day criteria for LtOT due to death. We also excluded those receiving a palliative/end-of-life care ICD-9$\mathrm{CM}^{30}$ (International Classification of Disease, 9th revision) diagnostic code on or before the opioid start date. The final analytic sample consisted of 17,044 patients.

\section{Indicators of Guideline-Concordant Care}

The independent variables of interest were indicators of guideline concordance derived from national clinical practice guidelines ${ }^{3,7-9}$ for the management of LtOT related to receipt of primary care visits, urine drug testing, psychotherapeutic cointerventions, rehabilitative therapies (i.e., occupational, physical, and rehabilitation therapies), and benzodiazepine co-prescriptions. Among patients with a current substance use disorder (SUD), we also examined receipt of inpatient or outpatient SUD treatment. In Table 1, we provide the operational definition for each indicator.

The decision regarding which indicators to examine was based on group consensus, relevance of indicator to patient safety, and accessibility of data in the electronic medical record system. Further details on the process and rationale for the inclusion of selected indicators can be found in a previous publication. ${ }^{16}$

Surveillance for guideline concordance began at the same time for all patients (LtOT start date) and continued for 180 days; this period was chosen because the initial months of LtOT represent a period during which the risks for adverse events are particularly high. ${ }^{3}$ For patients not completing 180 days of LtOT, the surveillance ended (either through death or censoring) when the opioid exposure ended, which was determined by either the opioid prescription stop date or, when applicable, date of death. Differences in the length of eligibility (i.e., LtOT duration) for receipt of indicators (opioid therapy duration) were accounted for in the analysis using timedependent methods (see "Statistical Analyses"). ${ }^{31,32}$

\section{Primary Outcome}

The outcome of interest was all-cause mortality. ${ }^{33}$ Consistent with our goal of understanding the association between guideline-concordant care and mortality in a diverse patient population, we examined this outcome for the sample in its entirety, and not stratified by particular patient sub-populations (e.g., HIV-infected). Specifically, we identified all patient deaths occurring in the 12-month period that began once patients completed the first 90 days of LtOT. Data on patient deaths were obtained from the Beneficiary Identification Records Locator Subsystem Death File, which was obtained from the Veterans Health Administration Vital Status File; these data are comparable to the National Death Index in terms of accuracy and completeness. ${ }^{34,35}$ 


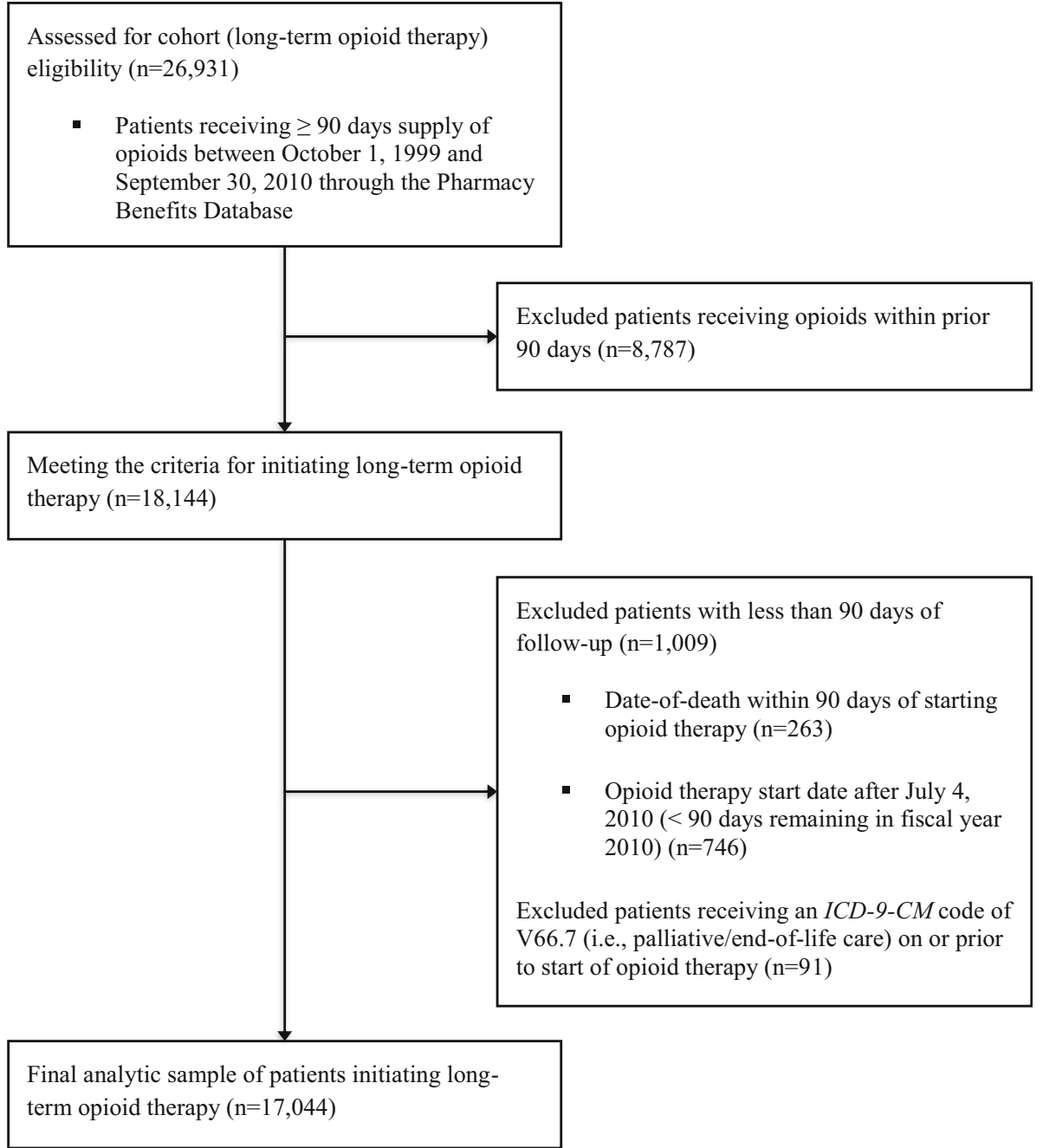

Figure 1 Study flow diagram.

\section{Covariates}

We used data from the VA National Patient Care Database $^{36}$ to characterize the sample at baseline, and used ICD-9-CM codes, pharmacy data, and laboratory results, when applicable, to describe clinical characteristics. A diagnosis of an alcohol use disorder was based on AUDIT-C (Alcohol Use Disorders Identification TestConsumption) scores $\geq 4$ or ICD-9-CM codes. Administrative codes were used to identify treatment. As a measure of overall severity of illness, we used the VACS Index, which incorporates age, HIV-1 RNA viral load, CD4 count, hemoglobin, FIB-4, estimated glomerular filtration rate, and hepatitis $\mathrm{C}$ virus. The VACS Index is a validated prognostic measure, ${ }^{28,29,37-40}$ which is predictive of morbidity and mortality in both HIVinfected and uninfected patients (in calculating the index, the assumption is made that uninfected patients have a CD4 count $>500$ cells $/ \mu \mathrm{L}$ and viral load $<20$ copies $/ \mathrm{ml}){ }^{29,41}$ Higher scores are indicative of higher all-cause mortality risk.

\section{Statistical Analyses}

Descriptive statistics were used to characterize the sample at baseline and according to receipt of guideline-concordant care. Differences according to demographic/clinical characteristics and treatment status were assessed with $\chi^{2}$ tests for categorical variables and, as appropriate, $t$, ANOVA, or Wilcoxon ranksum tests for continuous variables.

To control for confounding by indication, ${ }^{26,27}$ we generated propensity scores to reflect a patient's conditional probability of receiving the treatment of interest (i.e., guidelineconcordant care). Specifically, for each indicator, we developed a multivariable logistic regression model that included clinical covariates associated with both the treatment of interest and the outcome. These covariates were chosen from an extensive pool of mental health, major medical, and pain comorbidities that are prevalent in this study population, including major depression, bipolar disorder, post-traumatic stress disorder, psychosis, schizophrenia, schizoaffective disorder, alcohol and drug use disorders, HIV, hepatitis C, and diabetes. Pain comorbidities included those for acute pain 
Table 1 Operational Definitions for Indicators of Guideline-Concordant Long-Term Opioid Therapy

\begin{tabular}{|c|c|c|}
\hline Guideline indicators & Operational definition(s) & Source \\
\hline $\begin{array}{l}\text { Clinicians should conduct a follow-up visit within } 2-4 \text { weeks } \\
\text { of LTOT initiation. This initial phase should be considered a } \\
\text { therapeutic trial, for which opioid-naïve patients* are particularly } \\
\text { at risk. }{ }^{\dagger} \text { Clinicians should routinely reassess all patients on LtOT } \\
\text { every } 1-6 \text { months for risks and benefits of treatment for } \\
\text { duration of LtOT }\end{array}$ & $\begin{array}{l}\text { 1. Any documented outpatient PCP visit between } \\
\text { LtOT start date and end of } 180 \text { days of LtOT } \\
\text { (or LtOT stop date for patients on LtOT }<6 \text { months). }\end{array}$ & $\begin{array}{l}\text { APS/ } \\
\text { AAPM } \\
\text { VA/DoD } \\
8,9\end{array}$ \\
\hline \multicolumn{3}{|l|}{ Co-prescription of high-risk medications } \\
\hline Clinicians should avoid co-prescription of sedatives and LtOT. & $\begin{array}{l}\text { 3. Pharmacy documentation that patient was prescribed } \\
\text { benzodiazepines ( } \geq 7 \text { days so as to exclude prescriptions } \\
\text { for acute indications [e.g., pre-operative sedation]), } \\
\text { carisoprodol, or barbiturates between LtOT start date } \\
\text { and end of } 180 \text { days of LtOT (or LtOT stop date). }\end{array}$ & $\mathrm{VA} / \mathrm{DoD}^{8,9}$ \\
\hline \multicolumn{3}{|l|}{ Chronic pain co-interventions } \\
\hline \multirow{4}{*}{$\begin{array}{l}\text { Clinicians should avoid relying exclusively on opioids } \\
\text { for the management of chronic pain, and should } \\
\text { routinely take a multidisciplinary approach to pain } \\
\text { management that includes the integration of non-opioid } \\
\text { pharmacotherapies, rehabilitation or functional restoration, } \\
\text { and psychotherapeutic interventions. }\end{array}$} & 5. Physical rehabilitation therapies: & \multirow{4}{*}{$\begin{array}{l}\text { APS/ } \\
\text { AAPM } \\
\text { VA/DoD }\end{array}$} \\
\hline & Any documented outpatient visits to a VA physical & \\
\hline & $\begin{array}{l}\text { therapy, occupational therapy, or rehabilitation clinic } \\
\text { anytime between LtOT start date and end of } 180 \text { days }\end{array}$ & \\
\hline & 6. Psychotherapeutic co-interventions: & \\
\hline
\end{tabular}

Abbreviations: AAPM; American Academy of Pain Medicine; APS, American Pain Society; DoD, Department of Defense; LtOT, long-term opioid therapy; PCP, primary care provider; SUD, substance use disorder; UDT, urine drug test; VA, Veterans Administration.

*All patients in the current study were considered opioid-naïve (i.e., incident LtOT patients).

${ }^{\circ}$ Only the VA/DoD guidelines specify an exact time period.

"Lifetime history.

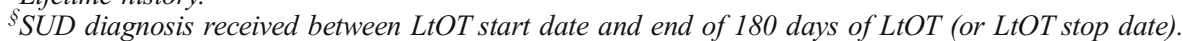

(e.g., abdominal or chest) and chronic pain (e.g., headache, back pain, arthritis, neuropathy), as previously described. ${ }^{6} \mathrm{We}$ also identified other covariates as potential confounders, including those for cardiac, pulmonary, liver, and renal disease. Additional covariates were then added to the models as necessary to adjust for receipt of other LtOT indicators (we demonstrated in previous analyses ${ }^{16}$ that HIV-infected patients are more likely to receive guideline-concordant care for certain indicators), opioid dose and schedule (for Schedule II, categorized as short- vs. long-acting), and to balance demographic and clinical characteristics. Finally, interactions were included to improve the fit of the model. C-statistics, a measure of a model's ability to predict patient treatment exposure status (i.e., discrimination), and goodness-of-fit tests (i.e., calibration) were used to evaluate the logistic regression models. Additional diagnostics included an evaluation of histograms (stratified by treatment status) for the propensity scores and plotting the observed versus expected outcomes from the Hosmer-Lemeshow goodness-of-fit tests. All logistic regression models were found to have good fit (HosmerLemeshow $p$ value $\geq .05)^{42,43}$ and acceptable $(0.75-0.80)$ to excellent $(0.80-0.90)^{44} \mathrm{c}$-statistics.

For each indicator, we then matched patients on propensity scores using a SAS greedy algorithm macro, ${ }^{45}$ designed to match on as many as five digits. For example, a patient with a low (or high) probability of receiving treatment was matched to another patient with a similar probability, yet only one of these patients received the treatment. The difference in the outcome (e.g., time to death) between these patients (or groups of patients) could then be attributed to the treatment, based on the covariates we included in the regression models. Further details on the methods and rationale for propensity-score matching in observational research are available elsewhere. ${ }^{26,46}$

Once 1:1 matching was completed, we then assessed for associations between guideline-concordant care and mortality using Cox proportional hazards regression with time-updated covariates. Time-updated methods were employed to account 
for differences in exposure to long-term opioids in cases where patients died or discontinued opioids prior to 180 days of therapy, and thus had less time to receive guidelineconcordant treatment (i.e., indicators). Such methods correctly classify patients' time to receipt (determined by variables reflecting initiation date) of a particular indicator as unexposed; once the indicator is obtained, the patient is thereafter classified as exposed. Upon a patient's death, proper estimates can then be obtained because the treatment status of those in the risk set has been properly classified. ${ }^{31,32}$

In secondary analyses, in addition to matching patients based on propensity scores, we examined the association between each of the indicators and mortality by adjusting ${ }^{47}$ with the propensity scores using both individual five-digit scores and scores categorized into quintiles (i.e., five strata). ${ }^{4-49}$ Overall, the results were similar whether we used the propensity scores for matching or regression-adjustment; ${ }^{47}$ thus, we present here the unmatched and propensity-matched results.

We conducted a sensitivity analysis specific to primary care visits to determine whether there was an association between mortality and receipt of multiple visits (i.e., 0 vs. $\geq 2$ visits; 0 vs. $\geq 3$ visits), for which we found no association. Thus, we present the results from the main analysis.

All analyses were performed using SAS software, version 9.4 (SAS Institute, Cary, NC, USA). A two-sided statistical significance level of 0.05 was applied to all analyses.

\section{RESULTS}

We identified 17,044 patients who initiated LtOT between 2000 and 2010. Patients were primarily male (98\%), of white $(47 \%)$ or black $(43 \%)$ race, with an average age (SD) of 50.2 (9.3) years (Table 2). In general, prior to matching on propensity scores, patients receiving each of the indicators were slightly older, HIV- and HCV-infected, and with a diagnosis of diabetes, serious mental illness, or SUD. These patients also had a higher mean VACS Index, indicating higher all-cause mortality risk. For the matched samples, select demographic and clinical characteristics according to each of the LtOT indicators can be found in Table 3 and in online Appendices 1A and 1B.

\section{Receipt of Guideline-Concordant Care}

In the 180 days following LtOT initiation, $86 \%$ of patients received a primary care visit, $20 \%$ a urine drug test, $32 \%$ psychotherapeutic co-interventions, and 30\% rehabilitative therapies. Co-prescriptions for benzodiazepines were received by $21 \%$ of patients, and receipt did not differ according to SUD history ( $22 \%$ vs. $22 \%$ for those with and without SUDs; $p=0.41)$ or current SUD status $(21 \%$ vs. $21 \% ; p=0.89)$. Among those with a current SUD, $45 \%$ were engaged in SUD treatment.

\section{Guideline-Concordant Care and All-Cause Mortality}

Unmatched Analyses. During 1 year of follow-up, there were 1048 (6\%) deaths, with a median (interquartile range [IQR]) time to death of 227.5 (154.0-328.5) days. In unadjusted analyses on an unmatched sample (Table 3), we found that psychotherapeutic cointerventions (hazard ratio [HR] 0.57; 95\% confidence interval [CI] $0.49-0.66 ; P<0.001)$ and rehabilitative therapies (HR $0.60 ; 95 \%$ CI $0.52-0.70 ; P<0.001$ ) were associated with a decrease in mortality, whereas benzodiazepine co-prescribing was associated with an increase in mortality (HR 1.63; 95\% CI 1.43-1.86; $P<0.001$ ). Among patients with a current SUD, SUD treatment was also associated with a decrease in mortality (HR 0.38;

Table 2 Patient Characteristics at Baseline

\begin{tabular}{|c|c|}
\hline Characteristic & $\begin{array}{l}\text { Overall sample } \\
(n=17,044)\end{array}$ \\
\hline Age, mean (SD), years & $50.2(9.3)$ \\
\hline Male Sex, $n(\%)$ & $16,638(98)$ \\
\hline \multicolumn{2}{|l|}{ Race, $n(\%)$} \\
\hline White & 7976 (47) \\
\hline Black & $7390(43)$ \\
\hline Hispanic & $1164(7)$ \\
\hline Other & $514(3)$ \\
\hline $\mathrm{HIV}, n(\%)$ & $5236(31)$ \\
\hline Hepatitis C, $n(\%)$ & $4809(28)$ \\
\hline Diabetes, $n(\%)$ & $5080(30)$ \\
\hline BMI, mean (SD) & $28.4(6.4)$ \\
\hline \multicolumn{2}{|l|}{ Smoking status, $n(\%)$} \\
\hline Never & $3548(22)$ \\
\hline Current & $10,269(63)$ \\
\hline Former & $2508(15)$ \\
\hline Chronic pain, $n(\%)^{\mathrm{a}}$ & $10,073(59)$ \\
\hline Acute pain, $n(\%)$ & 2306 (14) \\
\hline Major depression, $n(\%)$ & 3221 (19) \\
\hline Bipolar disorder, $n(\%)$ & $1757(10)$ \\
\hline PTSD, $n(\%)$ & $2797(16)$ \\
\hline Psychosis, $n(\%)$ & $1613(9)$ \\
\hline Active substance use disorder, $n(\%)$ & $3329(19.5)$ \\
\hline VACS Index, median (IQR) & $18.0(11.0-34.0)$ \\
\hline $\begin{array}{l}\text { Average daily opioid dose, mean (SD), mg } \\
\text { MEQ }\end{array}$ & $39.7(139.8)$ \\
\hline $\begin{array}{l}\text { Average daily opioid dose, median (IQR), } \\
\text { mg MEQ }^{\text {c }}\end{array}$ & $15.7(9.5-30.9)$ \\
\hline $\begin{array}{l}\text { Long-term opioid therapy duration, mean } \\
\text { (SD), days }\end{array}$ & $233.3(105.6)$ \\
\hline $\begin{array}{l}\text { Long-term opioid therapy duration, median } \\
\text { (IQR), days }\end{array}$ & $205.0(134.5-365.0)$ \\
\hline CD4 count, median (IQR), cells $/ \mu \mathrm{L}^{\mathrm{e}}$ & $357.0(182.0-567.0)$ \\
\hline $\begin{array}{l}\text { HIV-1 RNA, } \log _{10} \text { viral load, }<500 \text { copies/ } \\
\mathrm{ml}, n(\%)\end{array}$ & $2174(58)$ \\
\hline
\end{tabular}

Abbreviations: BMI, body mass index; IQR, interquartile range; PTSD, post-traumatic stress disorder; MEQ, morphine equivalent; VACS, Veterans Aging Cohort Study.

${ }^{a}$ Chronic pain: ICD-9-CM codes for headache, temporomandibular disorder, neck, back, extremity, arthritis, neuropathy, other.

${ }^{b}$ Acute pain: ICD-9-CM codes for abdominal, chest, fracture, kidney stones.

${ }^{c}$ Average daily opioid dose calculated by dividing the total morphine equivalents received in the year since starting long-term opioid therapy by total days' supply.

${ }^{d}$ During first year of long-term opioid therapy.

${ }^{e}$ Among HIV-infected patients. 
Table 3 Patient Characteristics According to Treatment Receipt in Matched Samples: Primary Care Visits and Urine Drug Tests

\begin{tabular}{|c|c|c|c|c|c|c|}
\hline \multirow[t]{2}{*}{ Characteristic } & \multicolumn{3}{|c|}{ Primary care visit $^{*}$} & \multicolumn{3}{|c|}{ Urine drug testing ${ }^{*}$} \\
\hline & No $(n=2341)$ & Yes $(n=2341)$ & $P$ value & No $(n=2579)$ & Yes $(n=2579)$ & $P$ value \\
\hline Age, mean (SD), years & $49.6(10.0)$ & $49.7(10.0)$ & 0.43 & $49.4(7.9)$ & $49.4(8.1)$ & 0.56 \\
\hline Male sex, $n(\%)$ & $2250(96)$ & $2244(96)$ & 0.66 & $2523(98)$ & $2518(98)$ & 0.64 \\
\hline Race, $n(\%)$ & & & 0.76 & & & 0.98 \\
\hline White & $1146(49)$ & $1145(49)$ & & $1025(40)$ & $1013(39)$ & \\
\hline Black & $971(41)$ & $986(42)$ & & $1351(52)$ & $1357(53)$ & \\
\hline Hispanic & $135(6)$ & $119(5)$ & & $147(6)$ & $151(6)$ & \\
\hline Other & $89(4)$ & $91(4)$ & & $56(2)$ & $58(2)$ & \\
\hline HIV, $n(\%)$ & 297 (13) & $310(13)$ & 0.57 & $893(35)$ & $896(35)$ & 0.93 \\
\hline Hepatitis C, $n(\%)$ & $512(22)$ & $526(22)$ & 0.62 & $1063(41)$ & $1047(41)$ & 0.65 \\
\hline Diabetes, $n(\%)$ & $585(25)$ & $574(25)$ & 0.71 & $677(26)$ & $673(26)$ & 0.90 \\
\hline BMI, mean (SD) & $28.4(6.1)$ & $29.0(6.8)$ & 0.07 & $27.7(6.2)$ & $27.5(6.1)$ & 0.54 \\
\hline Smoking status, $n(\%)$ & & & 0.84 & & & 0.01 \\
\hline Never & $517(24)$ & $531(24)$ & & $422(17)$ & $354(14)$ & \\
\hline Current & $1360(62)$ & $1356(62)$ & & $1735(70)$ & $1853(74)$ & \\
\hline Former & $321(15)$ & 311 (14) & & 324 (13) & $302(12)$ & \\
\hline Chronic pain, $n(\%)$ & $893(38)$ & 909 (39) & 0.63 & $1806(70)$ & $1763(68)$ & 0.19 \\
\hline Acute pain, $n(\%)$ & $210(9)$ & $226(10)$ & 0.42 & 451 (17) & 487 (19) & 0.19 \\
\hline Major depression, $n(\%)$ & $365(16)$ & $388(17)$ & 0.36 & $614(24)$ & $641(25)$ & 0.38 \\
\hline Bipolar disorder, $n(\%)$ & $236(10)$ & $229(10)$ & 0.73 & $374(15)$ & $376(15)$ & 0.94 \\
\hline PTSD, $n(\%)$ & $369(16)$ & 387 (17) & 0.47 & $512(20)$ & $522(20)$ & 0.73 \\
\hline Psychosis, $n(\%)$ & $211(9)$ & $227(10)$ & 0.42 & $315(12)$ & $338(13)$ & 0.34 \\
\hline Alcohol use disorder, $n(\%)$ & $626(27)$ & $669(29)$ & 0.16 & $1200(47)$ & $1173(45)$ & 0.45 \\
\hline Drug use disorder, $n(\%)$ & $478(20)$ & $500(21)$ & 0.43 & $1155(45)$ & $1118(43)$ & 0.30 \\
\hline VACS Index, median (IQR) & $15.0(6.0-27.0)$ & $16.0(10.0-28.0)$ & 0.71 & $22.0(12.0-35.0)$ & $22.0(11.0-35.0)$ & 0.78 \\
\hline $\begin{array}{l}\text { Average daily opioid dose, } \mathrm{mg} \mathrm{mEq} \text {, } \\
\text { median (IQR) }\end{array}$ & $14.8(9.0-28.1)$ & $14.4(8.7-26.7)$ & 0.42 & $18.6(10.6-43.1)$ & $18.6(10.8-40.5)$ & 0.81 \\
\hline
\end{tabular}

Abbreviations: BMI, body mass index; IQR, interquartile range; PTSD, post-traumatic stress disorder; MEQ, morphine equivalent; VACS, Veterans Aging Cohort Study.

*Percent of overall sample $(n=17,044)$ matched: primary care visit, $27.4 \%$; urine drug test, $30.2 \%$.

Note: Results for the remaining indicators can be found in online APPENDIX 1 A (Psychotherapeutic Co-Interventions and Rehabilitative Therapies) and online APPENDIX 1B (Benzodiazepine Co-Prescriptions and Substance Use Disorder Treatment).

95\% CI 0.29-0.50; $P<0.001)$. We did not detect an association between mortality and receipt of either primary care visits (HR 1.08; 95\% CI $0.91-1.28 ; P=0.37$ ) or urine drug testing (HR 1.10; 95\% CI 0.95-1.28; $P=0.20$; Table 4).

Propensity-Matched Analyses. As shown in Table 3 and in online Appendices $1 \mathrm{~A}$ and $1 \mathrm{~B}$, the propensity-matched samples were well balanced on demographic characteristics as well as medical, mental health, and SUD comorbidities. These matched samples support the associations from the unmatched analyses: psychotherapeutic co-interventions (HR 0.62; 95\% CI 0.51-0.75; $P<0.001$ ) and rehabilitative therapies (HR 0.81; 95\% CI $0.67-$ $0.98 ; P=0.03$ ) were associated with a decrease in mortality, whereas benzodiazepine co-prescribing was associated with an increase in mortality (HR 1.39; 95\% CI 1.12-1.66; $P<0.001$ ). Among patients with a current SUD, engagement in SUD treatment was also associated with a decrease in mortality (HR 0.47; $95 \%$ CI $0.32-0.68 ; P<0.001$ ). For primary care visits (HR 1.12; 95\% CI $0.90-1.26 ; P=0.32$ ) and urine drug tests (HR 0.96; 95\% CI $0.78-1.17 ; P=0.67)$, there remained no significant associations (Table 4 and Fig. $2 \mathrm{a}-\mathrm{c}$ ).

Table 4 Cox Proportional Hazards with Time-Updated Analyses of Time to Death According to Treatment Status in Unmatched and Propensity-Matched Patients

\begin{tabular}{|c|c|c|c|c|}
\hline \multirow[b]{2}{*}{ Indicator } & \multicolumn{2}{|l|}{ Unmatched $^{*}$} & \multicolumn{2}{|l|}{ Matched $^{\dagger}$} \\
\hline & $\begin{array}{l}\text { Hazard ratio } \\
(95 \% \mathrm{CI})\end{array}$ & $P$ value & $\begin{array}{l}\text { Hazard ratio } \\
(95 \% \mathrm{CI})\end{array}$ & $P$ value \\
\hline Primary care visit & $1.08(0.91-1.28)$ & 0.37 & $1.12(0.90-1.40)$ & 0.32 \\
\hline Urine drug testing & $1.10(0.95-1.28)$ & 0.20 & $0.96(0.78-1.17)$ & 0.67 \\
\hline Psychotherapeutic co-interventions & $0.57(0.49-0.66)$ & $<0.001$ & $0.62(0.51-0.75)$ & $<0.001$ \\
\hline Rehabilitative therapies & $0.60(0.52-0.70)$ & $<0.001$ & $0.81(0.67-0.98)$ & 0.03 \\
\hline $\begin{array}{l}\text { Benzodiazepine } \\
\text { co-prescribing }\end{array}$ & $1.63(1.43-1.86)$ & $<0.001$ & $1.39(1.12-1.66)$ & $<0.001$ \\
\hline Substance use disorder treatment & $0.38(0.29-0.50)$ & $<0.001$ & $0.47(0.32-0.68)$ & $<0.001$ \\
\hline
\end{tabular}

*Unmatched samples: $N=17,044$ for all treatments except substance use disorder treatment $(n=3329)$.

'Propensity-matched samples: primary care visit, $n=4682$; urine drug testing, $n=5158$; psychotherapeutic co-interventions, $n=7592 ;$ rehabilitative therapies, $n=9222$; benzodiazepine co-prescriptions, $n=6756$; substance use disorder treatment, $n=1656$. 
a

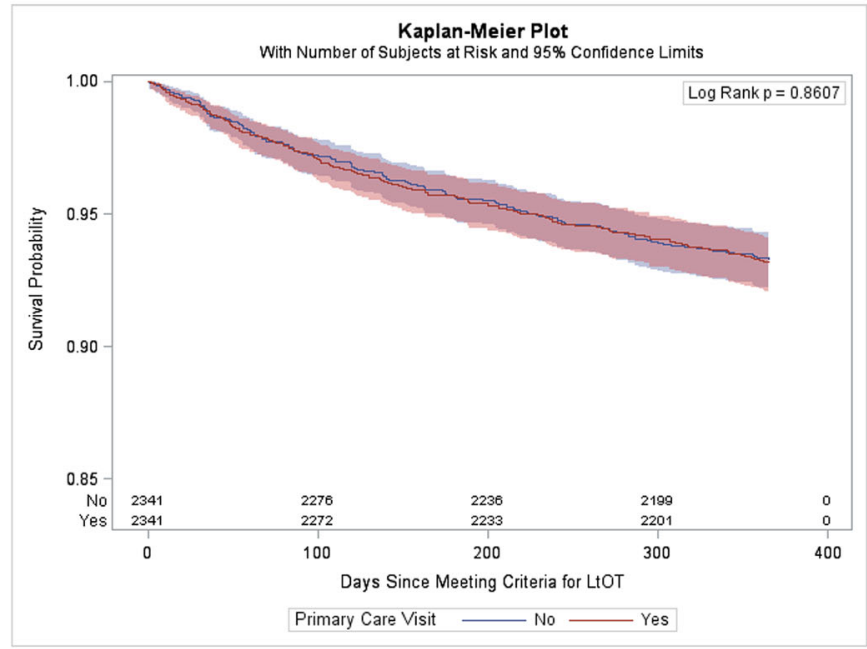

b

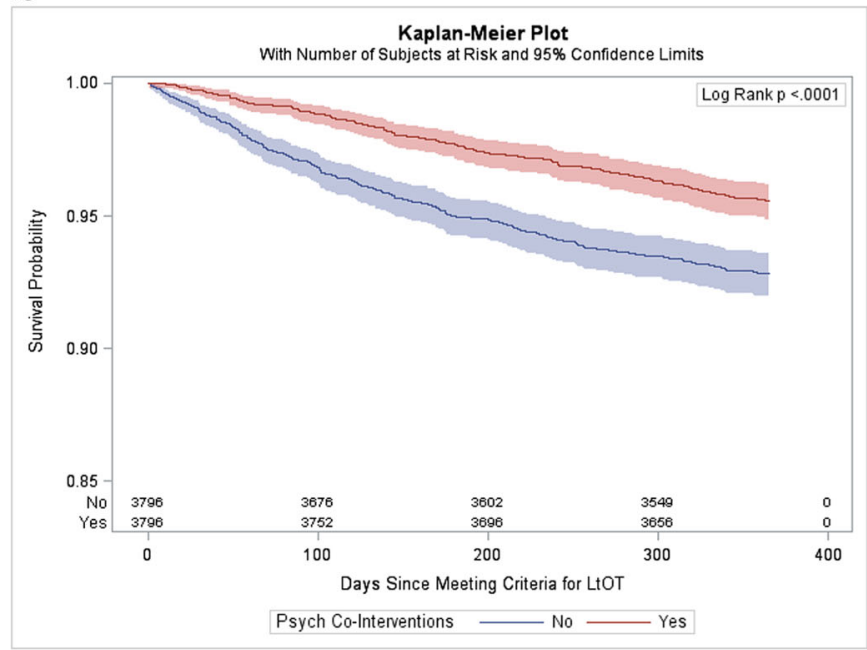

C

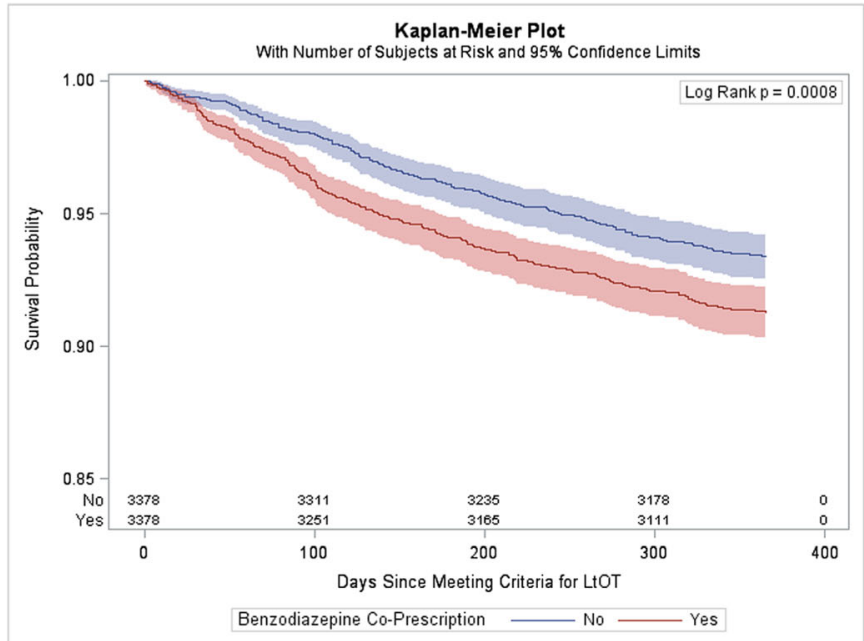

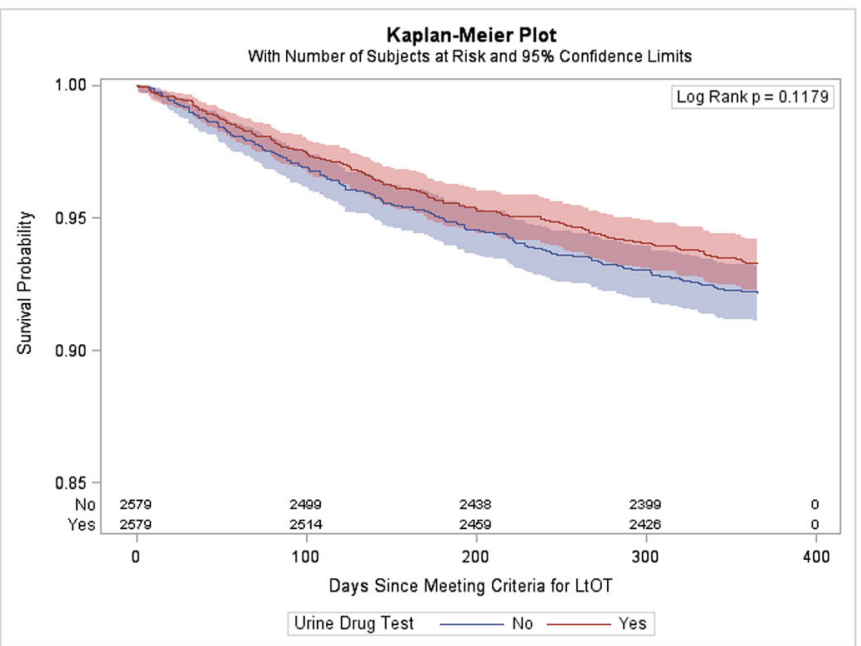
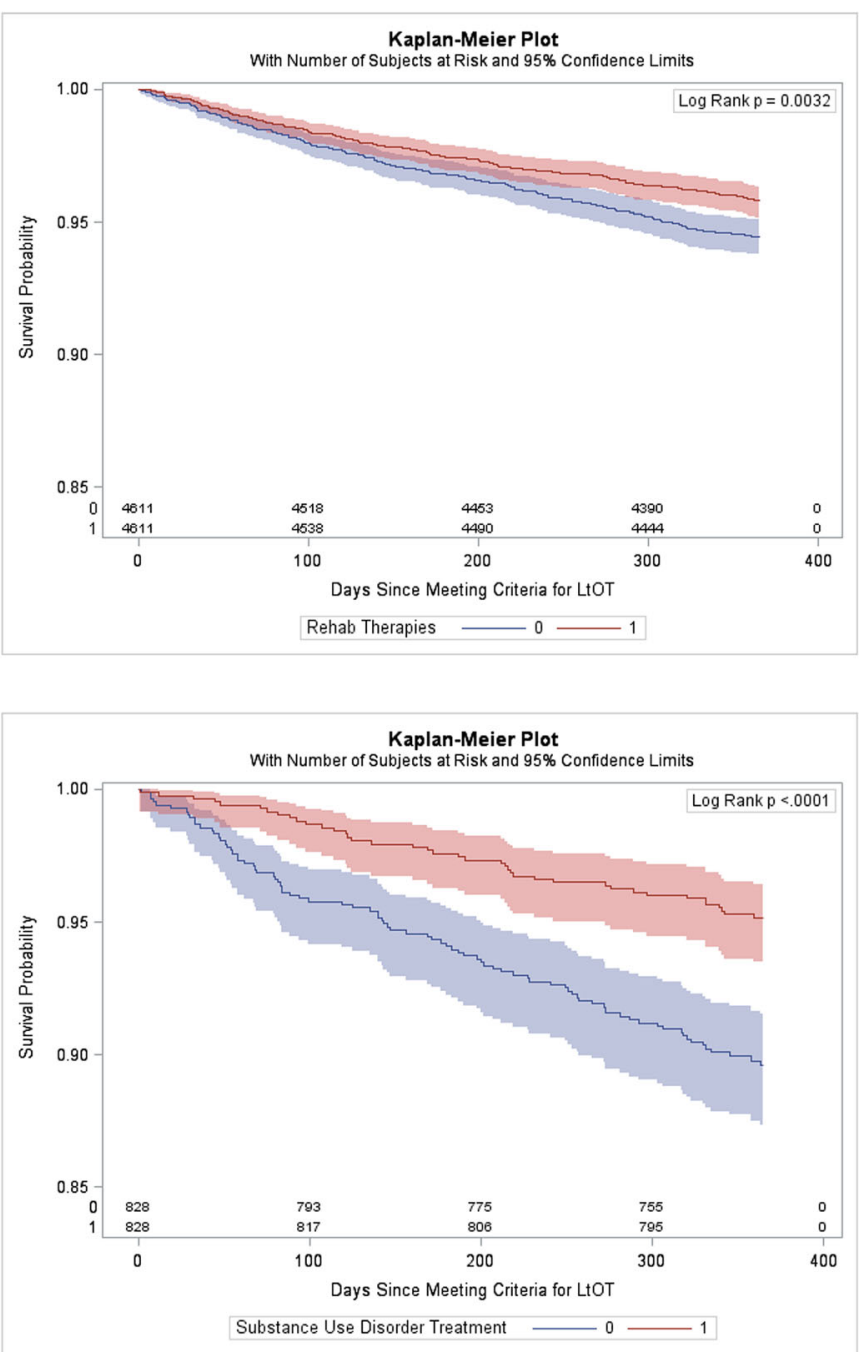

Figure 2 a Propensity-matched Kaplan-Meier plots for time to death (with number of patients at risk) according to treatment status: primary care visits (part 1) and urine drug tests (part 2). b Propensity-matched Kaplan-Meier plots for time to death (with number of patients at risk) according to treatment status: psychotherapeutic co-interventions (part 1) and rehabilitative therapies (part 2). c Propensity-matched KaplanMeier plots for time to death (with number of patients at risk) according to treatment status: benzodiazepine co-prescriptions (part 1) and substance use disorder treatment (part 2). 


\section{DISCUSSION}

Among 17,044 patients initiating LtOT between 2000 and 2010, $1048(6 \%)$ died during 1 year of follow-up. In this sample of patients with a high comorbidity burden, including HIV infection, and high rates of medical, psychiatric, and pain comorbidities, we found that, after extensively controlling for potential confounders, patients receiving psychotherapeutic co-interventions had an all-cause mortality rate nearly half that of patients not receiving these services. Moreover, patients receiving rehabilitative therapies during this same period were approximately $20 \%$ less likely to die from any cause than those not engaged in such treatment. Conversely, patients prescribed benzodiazepines concurrent with LtOT were approximately 1.5 times more likely to die. For patients with an untreated SUD, the risk of death was more than twice that of patients engaged in SUD treatment. We did not detect an association between primary care provider visits and all-cause mortality risk. Similarly, we did not detect an association between urine drug testing and mortality. This finding is in keeping with previous studies that have failed to demonstrate an impact of urine drug testing on opioid misuse, ${ }^{50,51}$ an indication that clinicians may not be acting on aberrant results. Indeed, for LtOT guidelines in general, research has shown that clinicians have been slow to integrate recommendations into patient care for those receiving LtOT, even for those at risk for opioid misuse and abuse, ${ }^{10-14}$ all of which speaks to the need ${ }^{52,53}$ for strategies to assist clinicians in caring for patients receiving LtOT. Provider training, in particular, is needed to support clinicians in responding to evidence of unsafe opioid use (e.g., aberrant urine toxicology). ${ }^{54}$

This observational study - the first to examine the association between guideline-concordant LtOT and mortality - lends support to current guidelines that promote a multidisciplinary approach to pain management, as well as to recommendations that caution against initiating LtOT in conjunction with sedatives and untreated SUDs. $^{3,7-9}$ Specifically, the guidelines encourage clinicians caring for patients receiving LtOT to routinely integrate interventions that target biological, psychological, and functional needs. ${ }^{3,7-9}$ Furthermore, for patients with a history of mental health or substance use disorders, the guidelines strongly recommend that clinicians consider co-managing these patients with specialists in mental health or addiction medicine, and according to the VA guidelines, LtOT is contraindicated in patients with a current SUD who are not receiving SUD treatment. ${ }^{8,9}$

Our study has several limitations. First, although we used propensity score matching to address potential differences between patients who did and did not receive (or adhere to) recommended care, residual confounding may still affect our findings. Additionally, we examined a limited number of indicators related to guideline-concordant care. It may be that indicators beyond the scope of our data (e.g., controlled substance agreements) are associated with reduced mortality in LtOT. ${ }^{3,55}$ Furthermore, because we restricted our sample to patients prescribed 90 or more days of opioids, by definition, deaths occurring in the initial days of opioid exposure, when the risks for LtOT are thought to be highest, ${ }^{3}$ were not included in the analyses (these patients had not yet met the criteria for LtOT). There may be a stronger association between receipt of guideline-concordant care and mortality earlier on in treatment, with the mortality benefit diminishing thereafter. In addition, although we excluded patients receiving a palliative/end-of-life care diagnosis on or before initiating LtOT, it is plausible that some of the differences we found in mortality reflect differences in the ability or desire of seriously ill patients or providers to comply with guideline-concordant care. Yet, for each of the indicators examined, a review of baseline characteristics in the matched samples shows that patients who did and did not receive treatment were comparable in terms of severity of illness, even for measures that were not included in the models to establish propensity scores. Furthermore, it was beyond the scope of the current paper to examine other important outcomes that may result from guideline-concordant LtOT, such as the prevention of opioid use disorder or improvement in functional status, pain relief, or patient satisfaction. Importantly, the VA and other state and federal agencies have recently instituted policy and practice initiatives ${ }^{56}$ that may have resulted in improvements in care in the time since our study ended. Finally, with this study, we were interested in the overall impact of guideline-concordant care on mortality and not the differential impact on particular patient subpopulations (e.g., HIV-infected patients). By employing propensity-score matching, we were able to include a range of covariates in the logistic regression models, which is in keeping with our overall research question: understanding the association between guideline-concordant LtOT and all-cause mortality among a diverse group of patients, including those with a high comorbidity and mortality burden. Due to the challenges of external validity inherent in this and other observational research, ${ }^{57}$ we encourage other investigators to replicate our analyses in a variety of patient cohorts.

We opted to focus on all-cause mortality rather than overdose deaths, for several reasons. First, opioids act on a variety of biologic systems and are associated with myriad adverse effects, including cardiovascular, endocrine, immunologic, and gastrointestinal effects - overdose mortality is just one concern. ${ }^{25,33,58-64}$ Moreover, limitations in the basic clinical epidemiology of overdose deaths often results in misclassification of cause of death, ${ }^{65-67}$ which likely precludes an accurate assessment ${ }^{68,69}$ of the association between guideline-concordant LtOT and overdose death. In particular, authorities have noted wide variation in overdose reporting across jurisdictions: medical examiners, coroners, and other practitioners do not use uniform standards and case definitions in conducting surveillance for, or classifying, deaths from overdose. ${ }^{70}$ Finally, we believe caution is indicated when assigning a single cause of death based only on ICD-9 codes to patients with complex medical conditions (e.g., HIV) ${ }^{71}$

With this study, our aim was to contribute to the evidence base for clinical practice guidelines promulgated to improve quality of care for patients receiving LtOT. Further research, however, is needed to understand the impact on patient outcomes of targeted interventions ${ }^{56}$ that directly address opioid safety and efficacy, 
the optimal timing and frequency of such interventions, and patient subpopulations most likely to benefit. Experimental research, in particular, is needed to determine whether adherence to specific guidelines results in a decrease in mortality and other adverse events. Additional research is also needed to understand the role of guideline-concordant LtOT on cause-specific mortality, especially with regard to overdose deaths.

Our findings from this observational study suggest that adherence to select opioid clinical practice guidelines is associated with lower mortality among individuals initiating LtOT for chronic non-cancer pain. Patients may benefit from interdisciplinary care that extends beyond routine follow-up to encompass multi-modal treatment models - particularly SUD treatment, psychotherapeutic co-interventions, and rehabilitative therapies - that address chronic pain and its associated comorbidities across multiple disciplines.

Author Contributions: Dr. Gaither had full access to all the data in the study and takes responsibility for the integrity of the study and the accuracy of the data analysis.

Corresponding Author: Julie R. Gaither, PhD, MPH, RN; VA Connecticut Healthcare System, 950 Campbell Avenue, West Haven, CT 06516, USA (e-mail: julie.gaither@yale.edu).

\section{Compliance with ethical standards:}

Disclosure: The content of this paper is solely the responsibility of the authors and does not necessarily reflect the official views of the $\mathrm{Na}$ tional Institutes of Health or the Department of Veterans Affairs.

Conflict of Interest: Dr. Fiellin received an honorarium from PinneyAssociates to serve on an external advisory board to monitor the diversion and abuse of buprenorphine. All other authors declare that they do not have a conflict of interest.

Funding/Support: Research reported in this paper was supported by grants from the National Institute on Drug Abuse (F31DA035567; K12DA033312), National Institute on Alcohol Abuse and Alcoholism (U1OAA013566; U01AA020790; U24AA020794), National Institute of Mental Health (P3OMH062294), VA Health Services Research and Development Center of Innovation (CIN 13-047), and the Agency for Healthcare Research and Quality (U19HS21112). These organizations had no role in the design, conduct, or reporting of this study.

\section{REFERENCES}

1. Volkow ND, McLellan TA, Cotto JH, Karithanom M, Weiss SR. Characteristics of opioid prescriptions in 2009. JAMA. 2011;305(13):1299-1301.

2. Korff MV, Saunders K, Thomas Ray G, et al. De facto long-term opioid therapy for noncancer pain. Clin J Pain. 2008;24(6):521-527.

3. Chou R, Fanciullo GJ, Fine PG, et al. Clinical guidelines for the use of chronic opioid therapy in chronic noncancer pain. J Pain. 2009; 10(2):113130 .

4. Edlund MJ, Steffick D, Hudson T, Harris KM, Sullivan M. Risk factors for clinically recognized opioid abuse and dependence among veterans using opioids for chronic non-cancer pain. Pain. 2007;129(3):355-362.

5. Braden JB, Sullivan MD, Ray GT, et al. Trends in long-term opioid therapy for noncancer pain among persons with a history of depression. Gen Hosp Psychiatry. 2009;31(6):564-570.

6. Edelman EJ, Gordon $\mathbf{K}$, Becker WC, et al. Receipt of opioid analgesics by HIV-infected and uninfected patients. J Gen Intern Med. 2013;28(1):82-90.

7. American Pain Society/American Academy of Pain Medicine. The use of opioids for treatment of chronic pain. A consensus statement from the
American Academy of Pain Medicine and the American Pain Society. Clin J Pain. 1997;13:6-8.

8. US Department of Veterans Affairs/Department of Defense. Clinical Practice Guideline for Management of Opioid Therapy for Chronic Pain. 2010.

9. US Department of Veterans Affairs/Department of Defense. Clinical Practice Guideline for Management of Opioid Therapy for Chronic Pain. 2003.

10. Krebs EE, Ramsey DC, Miloshoff JM, Bair MJ. Primary care monitoring of long-term opioid therapy among veterans with chronic pain. Pain Med. $2011 ; 12(5): 740-746$.

11. Morasco BJ, Turk DC, Donovan DM, Dobscha SK. Risk for prescription opioid misuse among patients with a history of substance use disorder. Drug Alcohol Depend. 2013;127(1-3): 193-199.

12. Morasco BJ, Cavanagh R, Gritzner S, Dobscha SK. Care management practices for chronic pain in veterans prescribed high doses of opioid medications. Fam Pract. 2013;30(6):671-678.

13. Morasco BJ, Duckart JP, Dobscha SK. Adherence to clinical guidelines for opioid therapy for chronic pain in patients with substance use disorder. J Gen Intern Med. 2011;26(9):965-971.

14. Önen NF, Barrette EP, Shacham E, Taniguchi T, Donovan M, Overton ET. A review of opioid prescribing practices and associations with repeat opioid prescriptions in a contemporary outpatient HIV clinic. Pain Pract. 2012; 12(6):440-448.

15. Rasu RS, Sohraby R, Cunningham L, Knell ME. Assessing chronic pain treatment practices and evaluating adherence to chronic pain clinical guidelines in outpatient practices in the United States. J Pain. 2013;14(6):568-578.

16. Gaither JR, Goulet JL, Becker WC, et al. Guideline-Concordant Management of Opioid Therapy Among Human Immunodeficiency Virus (HIV)-Infected and Uninfected Veterans. J Pain. 2014;15(11):1130-1140.

17. Pronovost P. Enhancing physicians' use of clinical guidelines. JAMA. 2013;310(23):2501-2502.

18. Peppin JF, Passik SD, Couto JE, et al. Recommendations for urine drug monitoring as a component of opioid therapy in the treatment of chronic pain. Pain Med. 2012;13(7):886-896.

19. Chou R, Ballantyne JC, Fanciullo GJ, Fine PG, Miaskowski C. Research gaps on use of opioids for chronic noncancer pain: findings from a review of the evidence for an American Pain Society and American Academy of Pain Medicine clinical practice guideline. J Pain. 2009; 10(2): 147-159.

20. Chou R, Turner JA, Devine EB, et al. The Effectiveness and Risks of Long-Term Opioid Therapy for Chronic Pain: A Systematic Review for a National Institutes of Health Pathways to Prevention Workshop. Ann Intern Med. 2015.

21. Yale School of Medicine/Veteran Aging Cohort Study. http://medicine.yale. edu/intmed/vacs. Accessed October 27, 2015.

22. Justice AC, Dombrowski E, Conigliaro J, et al. Veterans Aging Cohort Study (VACS): Overview and description. Med Care. 2006;44(8 Suppl 2):S13-S24.

23. Justice AC, Erdos J, Brandt C, Conigliaro J, Tierney W, Bryant K. The Veterans Affairs Healthcare System: A unique laboratory for observational and interventional research. Med Care. 2006;44(8 Suppl 2):S7-S12.

24. Fultz SL, Skanderson M, Mole LA, et al. Development and verification of a "virtual" cohort using the National VA Health Information System. Med Care. 2006;44(8 Suppl 2):S25-S30.

25. Korff Mv. Long-term opioid therapy reconsidered. Ann Intern Med. 2011;155(5):325-328.

26. Joffe MM, Rosenbaum PR. Invited commentary: propensity scores. Am J Epidemiol. 1999;150(4):327-333.

27. Pasta DJ. Using Propensity Scores to Adjust for Group Differences: Examples Comparing Alternative Surgical Methods. Paper presented at: Twenty-Sixth Annual SAS Users Group International Conference, SAS Institute Inc., 2001; Cary, NC.

28. Justice AC, Modur SP, Tate JP, et al. Predictive accuracy of the Veterans Aging Cohort Study index for mortality with HIV infection: a North American cross cohort analysis. J Acquir Immune Defic. Syndr. 2013;62(2):149-163.

29. Yale School of Medicine/Veteran Aging Cohort Study. http://www. vacohort.org/welcome/vacsindexinfo.aspx. Accessed October 27, 2015.

30. Centers for Disease Control and Prevention. International Classification of Diseases, Ninth Revision, Clinical Modification. Hyattsville, MD: National Center for Health Statistics; 2010.

31. Suissa S. Immortal Time Bias in Obeservational Studies of Drug Effects. Pharmacoepidemiol Drug Saf. 2007;16(3):241-249.

32. Allison PD, SAS Institute. Survival analysis using the SAS system: a practical guide, second edition. Cary, NC: SAS Institute; 2010. 
33. Miller M, Barber CW, Leatherman S, et al. Prescription opioid duration of action and the risk of unintentional overdose among patients receiving opioid therapy. JAMA Internal Medicine. 2015;175(4):608-615.

34. Cowper DC, Kubal JD, Maynard C, Hynes DM. A primer and comparative review of major US mortality databases. Ann Epidemiol. 2002;12(7):462468.

35. Fisher SG, Weber L, Goldberg J, Davis F. Mortality ascertainment in the veteran population: alternatives to the National Death Index. Am J Epidemiol. 1995;141(3):242-250.

36. US Department of Veterans Affairs National Patient Care Database (NPCD). https://catalog.data.gov/dataset/national-patient-care-database-npcd. Accessed October 27, 2015.

37. Bebu I, Tate J, Rimland D, et al. The VACS Index Predicts Mortality in a Young, Healthy HIV Population Starting Highly Active Antiretroviral Therapy. J Acquir Immune Defic Syndr. 2014;65(2):226-230.

38. Justice AC, McGinnis KA, Skanderson M, et al. Towards a combined prognostic index for survival in HIV infection: the role of 'non-HIV biomarkers. HIV Med. 2010;11(2):143-151.

39. Tate JP, Justice AC, Hughes MD, et al. An internationally generalizable risk index for mortality after one year of antiretroviral therapy. AIDS. 2013;27(4):563-572.

40. Brown ST, Tate JP, Kyriakides TC, et al. The VACS index accurately predicts mortality and treatment response among multi-drug resistant HIV infected patients participating in the options in management with antiretrovirals (OPTIMA) study. PLoS One. 2014;9(3), e92606.

41. Akgun KM, Tate JP, Pisani M, et al. Medical ICU admission diagnoses and outcomes in human immunodeficiency virus-infected and virusuninfected veterans in the combination antiretroviral era. Crit Care Med. 2013;41(6): 1458-1467.

42. Allison PD, Institute SAS. Logistic regression using SAS : theory and application. 2nd ed. Cary, N.C: SAS Pub.; 2012

43. Hosmer DW, Lemeshow S. Applied logistic regression. Wiley series in probability and statistics Texts and references section. 2nd ed. New York: Wiley; 2000: http://dx.doi.org/10.1002/0471722146. Accessed October 27, 2015.

44. Schneeweiss S, Seeger JD, Maclure M, Wang PS, Avorn J, Glynn RJ. Performance of comorbidity scores to control for confounding in epidemiologic studies using claims data. Am J Epidemiol. 2001;154(9):854-864.

45. Parsons L. Reducing bias in a propensity score matched-pair sample using greedy matching techniques. Paper presented at: Twenty-Fifth Annual SAS Users Group International Conference, SAS Institute Inc., 2000; Cary, NC.

46. Rosenblum A, Marsch LA, Joseph H, Portenoy RK. Opioids and the treatment of chronic pain: controversies, current status, and future directions. Exp Clin Psychopharmacol. 2008;16(5):405-416.

47. D'Agostino RB Jr. Propensity score methods for bias reduction in the comparison of a treatment to a non-randomized control group. Stat Med. 1998;17(19):2265-2281.

48. Cochran WG. The effectiveness of adjustment by subclassification in removing bias in observational studies. Biometrics. 1968;24(2):295313.

49. Pasta DJ. Using Propensity Scores to Adjust for Group Differences: Examples Comparing Alternative Surgical Methods.

50. Starrels JL, Becker WC, Alford DP, Kapoor A, Williams AR, Turner BJ. Systematic review: treatment agreements and urine drug testing to reduce opioid misuse in patients with chronic pain. Ann Intern Med. 2010;152(11):712-720.

51. Turner JA, Saunders K, Shortreed SM, et al. Chronic opioid therapy risk reduction initiative: impact on urine drug testing rates and results. J Gen Intern Med. 2014;29(2):305-311
52. Lum PJ, Little S, Botsko M, et al. Opioid-prescribing practices and provider confidence recognizing opioid analgesic abuse in HIV primary care settings. J Acquir Immune Defic Syndr. 2011;56(Suppl 1):S91-S97.

53. Vijayaraghavan M, Penko J, Guzman D, Miaskowski C, Kushel MB. Primary care providers' judgments of opioid analgesic misuse in a community-based cohort of HIV-infected indigent adults. J Gen Intern Med. 2011;26(4):412-418.

54. Starrels JL, Fox AD, Kunins HV, Cunningham CO. They don't know what they don't know: internal medicine residents' knowledge and confidence in urine drug test interpretation for patients with chronic pain. J Gen Intern Med. 2012;27(11):1521-1527.

55. Chou R. 2009 Clinical Guidelines from the American Pain Society and the American Academy of Pain Medicine on the use of chronic opioid therapy in chronic noncancer pain: what are the key messages for clinical practice? Pol Arch Med Wewn. 2009;119(7-8):469-477.

56. US Department of Veterans Affairs. Office of Public and Intergovernmental Affairs. VA initiative shows early promise in reducing use of opioids for chronic pain [news release]. February 25, 2014. http://www.va.gov/opa/ pressrel/pressrelease. .fm?id=2529. Accessed October 27, 2015.

57. von Elm E, Altman DG, Egger M, et al. The Strengthening the Reporting of Observational Studies in Epidemiology (STROBE) statement: guidelines for reporting observational studies. Lancet. 2007;370(9596):1453-1457.

58. Veldhuizen S, Callaghan RC. Cause-specific mortality among people previously hospitalized with opioid-related conditions: a retrospective cohort study. Ann Epidemiol. 2014;24(8):620-624

59. Solomon DH, Rassen JA, Glynn RJ, et al. The comparative safety of opioids for nonmalignant pain in older adults. Arch. Intern. Med. 2010;170(22): 1979-1986.

60. Solomon DH, Rassen JA, Glynn RJ, Lee J, Levin R, Schneeweiss S. The comparative safety of analgesics in older adults with arthritis. Arch Intern Med. 2010;170(22):1968-1976.

61. Roy S, Ninkovic J, Banerjee S, et al. Opioid drug abuse and modulation of immune function: consequences in the susceptibility to opportunistic infections. J Neuroimmune Pharmacol. 2011;6(4):442-465.

62. Li L, Setoguchi S, Cabral H, Jick S. Opioid use for noncancer pain and risk of myocardial infarction amongst adults. J Intern Med. 2013;273(5):511-526

63. Saunders KW, Dunn KM, Merrill JO, et al. Relationship of opioid use and dosage levels to fractures in older chronic pain patients. J Gen Intern Med. 2010;25(4):310-315.

64. Dublin S, Walker RL, Jackson ML, et al. Use of opioids or benzodiazepines and risk of pneumonia in older adults: a population-based casecontrol study. J Am Geriatr Soc. 2011;59(10):1899-1907.

65. Slavova S, O'Brien DB, Creppage $\mathbf{K}$, et al. Drug Overdose Deaths: Let's Get Specific. Public Health Rep. 2015;130(4):339-342.

66. Goldberger BA, Maxwell JC, Campbell A, Wilford BB. Uniform standards and case definitions for classifying opioid-related deaths: recommendations by a SAMHSA consensus panel. J Addict Dis. 2013;32(3):231-243.

67. Huguet N, Kaplan MS, McFarland BH. The effects of misclassification biases on veteran suicide rate estimates. Am J Public Health. 2014; 104(1):151-155.

68. Betz ME, Kelly SP, Fisher J. Death certificate inaccuracy and underreporting of injury in elderly people. J Am Geriatr Soc. 2008;56(12):2267-2272.

69. Myers KA, Farquhar DR. Improving the accuracy of death certification. CMAJ. 1998;158(10):1317-1323.

70. Warner M, Paulozzi LJ, Nolte KB, Davis GG, Nelson LS. State variation in certifying manner of death and drugs involved in drug intoxification deaths. Acad Forensic Pathol. 2013;3(2):231-237.

71. Justice AC. Commentary: Treated HIV infection is a chronic disease: the case against cause of death analyses. Int J Epidemiol. 2010;39(1):146-148. 